\title{
Filmes plásticos e ácido ascórbico na qualidade de araticum minimamente processado
}

\author{
Plastic packaging film and ascorbic acid treatment on the quality of fresh cut araticum
}

\author{
Manoel Soares Soares Júnior ${ }^{I^{*}}$ Marcio Caliari ${ }^{\mathrm{I}}$ Rosângela Vera ${ }^{\mathrm{I}}$ \\ Camila Silveira Melo ${ }^{\text {II }}$
}

\section{RESUMO}

O objetivo deste trabalho foi avaliar os efeitos do ácido ascórbico e do tipo de filme plástico como embalagem na qualidade do araticum minimamente processado e mantido sob refrigeração. O ácido ascórbico não evitou o escurecimento do araticum minimamente processado. Independentemente do tipo de embalagem, a acidez titulável aumentou com o tempo. A embalagem de policloreto de vinila ou polietileno de baixa densidade promoveu uma significativa perda de massa se comparada com a a laminada a vácuo. A embalagem laminada a vácuo propiciou vida-de-prateleira mais longa ao produto, o qual permaneceu com aparência adequada $e$ qualidade comercial até o sétimo dia do armazenamento. A vida-de-prateleira dos demais tratamentos alcançou somente três dias.

Palavras-chave: Annona crassiflora Mart., processamento, embalagem, vida-de-prateleira.

\section{ABSTRACT}

This study was aimed at evaluating the effect of ascorbic acid and type of plastic packaging film on the quality of refrigerated fresh cut araticum. Ascorbic acid did not prevent fresh cut araticum from darkening. Regardless of the type of plastic packaging, the fruit titrable acidity increased with time. Packing with polyvynil chloride or with low density polyethylene promoted a significant mass loss compared to laminate vacuum packaging. Laminate vacuum packaging increased the shelf life of the product up 7 days, maintaining its commercial quality and appearance. The shelf life of the other treatments reached 3 days only.

Key words: Annona crassiflora Mart., processing, packaging, shelf life.

\section{INTRODUÇÃO}

O araticum (Annona crassiflora Mart.), também conhecido popularmente como bruto, cabeçade-negro, cascudo, marolo e pinha-do-cerrado, é uma espécie frutífera da família Annonaceae, assim como a cherimóia, fruta-do-conde, pinha, graviola e outras (RIBEIRO et al., 2000). Ocorre no cerrado da Região Centro-Oeste, em Tocantins, Minas Gerais e Bahia, fornecendo, em condições naturais, frutos maduros entre fevereiro e março, que são muito apreciados pela polpa doce, com aroma bastante forte. Apresenta casca de coloração amarelo-esverdeada, lisa ou recoberta por escamas carnosas e grande número de sementes (SILVA et al.,1997). Sua comercialização tem sido realizada às margens das rodovias, em alguns mercados e feiras regionais, com grande aceitação popular. Além de consumidos in natura, esses frutos podem ser transformados em sucos, sorvetes, licores, pães, bolos e deliciosas refeições (SILVA et al., 2001). Uma alternativa para a comercialização do araticum pode ser o processamento mínimo, com a disposição dos frutilhos descascados em bandejas de polietileno expandido, embaladas em filmes plásticos e sob refrigeração, em redes de supermercados e hipermercados dos grandes centros urbanos, como Goiânia, Anápolis e Brasília.

Frutos minimamente processados são definidos como produtos preparados por uma ou

\footnotetext{
'Departamento de Engenharia de Alimentos, Universidade Federal de Goiás (UFG), Campus Samambaia, 74001-970, Goiânia, GO, Brasil. E-mail: manoel@agro.ufg.br. *Autor para correspondência.

${ }^{\text {IIC }}$ urso de Engenharia de Alimentos, UFG, Goiânia, GO, Brasil.
} 
algumas unidades de operação apropriadas, tais como descascamento, fatiamento, corte, raspagem, retalhamento e outras, garantindo a qualidade e sanidade (WILEY, 1994). CHITARRA \& CHITARRA (2005) recomendam o uso da refrigeração para o armazenamento de vegetais minimamente processados, pois esta retarda o metabolismo, diminuindo a taxa respiratória e a atividade enzimática, evitando ou minimizando alterações no aroma, sabor, textura, cor e demais atributos de qualidade.

Na comercialização, a embalagem é um requisito essencial para a manutenção da qualidade para esse tipo de mercadoria, oferecendo uma possibilidade de estender a vida de armazenagem póscolheita, já que cria uma barreira que retarda a perda do sabor e do aroma desejáveis e do vapor de água, enquanto restringe a troca de $\mathrm{CO}_{2}$ e $\mathrm{O}_{2}$, criando uma atmosfera modificada (BALDWIN et al., 1995).

Antes de embalar o produto, pode-se fazer uso de aditivos químicos para aumentar a vida-deprateleira (CHITARRA, 1998). A utilização do ácido ascórbico pode inibir temporariamente o escurecimento enzimático de frutos minimamente processados, pois reage com o oxigênio atmosférico formando o ácido dehidroascórbico (ARAÚJO, 2001).

O objetivo deste trabalho foi avaliar o efeito do ácido ascórbico e do tipo de filme plástico utilizado como embalagem na qualidade do araticum minimamente processado sob armazenamento refrigerado.

\section{MATERIAL E MÉTODOS}

Os frutos provenientes de áreas de cerrado do Estado do Tocantins foram colhidos manualmente, quando se apresentavam no estágio "de vez", ou seja com pequenos sinais de abertura na casca (RIBEIRO et al., 2000). Em seguida, foram embalados em caixas de madeira tipo $\mathrm{K}$, transportados e armazenados sob temperatura ambiente e processados seis dias após a colheita, tempo necessário para que os frutos atingissem características sensoriais ideais, como aroma marcante e pronunciado.

O experimento foi conduzido na Escola de Agronomia e Engenharia de Alimentos da UFG, em Goiânia - GO, em março de 2004. Avaliaram-se os caracteres físicos de 15 frutos de araticum. Para tanto, esses foram numerados ao acaso, determinando-se a seguir o comprimento e os diâmetros longitudinal e transversal com paquímetro de precisão de $0,05 \mathrm{~mm}$, bem como a matéria fresca total, usando-se para isso uma balança de precisão de $0,01 \mathrm{~g}$.
Selecionaram-se os frutos mais maduros, ou seja, aqueles que apresentavam distância mais pronunciada entre os frutilhos, amolecimento da polpa e exalação forte de aroma característico (RIBEIRO et al., 2000), descartando-se os atacados por fungos, ou deformados. Os frutos selecionados foram submetidos a lavagens por imersão e em água corrente e sanitizados por imersão em solução de hipoclorito de sódio a 100mg $\mathrm{L}^{-1}$, durante 15 minutos. Logo após, as cascas foram retiradas manualmente em recipientes higienizados e os frutilhos foram destacados do eixo central.

As cascas, os pedúnculos e os eixos centrais foram pesados para avaliação do rendimento dos frutos.

$\mathrm{O}$ experimento foi composto por seis tratamentos: três filmes plásticos como embalagens, com e sem adição de ácido ascórbico. Aplicou-se o delineamento casualizado, com três repetições. Para realizar as análises físico-químicas, foi necessário confeccionar 108 amostras, pois, a cada dois dias, 18 amostras foram analisadas de forma destrutiva.

Os filmes utilizados foram: policloreto de vinila (PVC), marca Royalpack, com espessura de $15 \mu \mathrm{m}$; polietileno de baixa densidade (PE), marca Cosplastic, com espessura de $30 \mu \mathrm{m}$; e laminado $(\mathrm{PE}+$ Nylon $+\mathrm{PE})$, marca Cosvac MBB, destinado à embalagem a vácuo, com espessura de $75 \mu \mathrm{m}$. Nas amostras com laminado, utilizou-se vácuo parcial dentro das embalagens.

Bandejas de poliestireno expandido de $15 \mathrm{~cm}$ $\mathrm{x} 15 \mathrm{~cm}$ foram sanitizadas por imersão durante 15 minutos e deixadas no ambiente de processamento até secarem. Porções de aproximadamente $70 \mathrm{~g}$ de frutilhos, provenientes da mistura de oito frutos selecionados, foram distribuídas sobre essas bandejas.

Nas amostras com ácido ascórbico, uma solução de $3,5 \mathrm{~g}$ de ácido ascórbico por $100 \mathrm{~mL}$ de água destilada foi aspergida sobre os frutilhos por meio de um borrifador de forma a obter uma dosagem de $0,03 \mathrm{~g}$ de ácido por $100 \mathrm{~g}$ de frutilhos. O filme de PVC foi colocado manualmente ao redor de cada bandeja e imediatamente termosselado. Os sacos de PEBD e de laminados foram termossoldados em embaladora comum e a vácuo, respectivamente. Todas as amostras foram armazenadas em câmara fria sem controle da umidade relativa do ar a aproximadamente $11^{\circ} \mathrm{C}$.

Determinaram-se os atributos de qualidade durante um período de 10 dias. A perda de massa fresca (PMF) foi calculada utilizando-se a expressão: $\mathrm{PMF} \%=$ (MI-MS).100/MI, em que MI é massa inicial e MS a massa da amostra no dia da avaliação. O teor de sólidos solúveis (SS) foi determinado com um refratômetro Instrutherm modelo RT-30ATC, e o de acidez titulável (AT) por titulometria (INSTITUTO ADOLFO LUTZ, 1985). Esses teores foram utilizados no cálculo da 
relação SS/AT. O pH foi determinado por meio de um potenciômetro Análion, modelo PM 608. Determinouse também a percentagem de frutilhos atacados por microrganismos em relação ao número total de frutilhos por bandeja, ataque caracterizado pela visualização de manchas de coloração rosa. Para a avaliação da aparência, realizada diariamente até o $11^{\circ}$ dia, empregouse uma escala de notas de 1 a 4, adaptada de UNDURRAGA et al. (1997), segundo o qual a nota 1 expressa aparência muito boa, a nota 2 boa, a nota 3 regular e a 4 péssima.

A análise estatística dos dados obtidos compreendeu a realização de regressão para cada tratamento, em função dos dias de armazenamento, e teste de Tukey a $5 \%$ de probabilidade de erro para comparação das médias dos tratamentos (SAS, 1996). Somente a aparência foi avaliada através da estatística não-paramétrica, segundo o teste Kruskal-Wallis, que substitui o teste $\mathrm{F}$, e o teste de comparação de médias de ensaios inteiramente casualizados a $5 \%$ de probabilidade de erro, que substitui o teste de Tukey (GOMES, 1985). As respostas cujas dimensões foram dadas em percentagem sofreram transformação segundo a expressão: $y=$ arco $\operatorname{sen}(\mathrm{X} / 100) 1 / 2$, para possibilitar a aplicação da ANOVA e do teste de Tukey.

\section{RESULTADOS E DISCUSSÃO}

As médias do comprimento, diâmetro longitudinal, diâmetro transversal dos frutos em posição de repouso (pedúnculo paralelo ao plano) e da massa da matéria fresca foram de 14,0 $02,2 \mathrm{~cm}, 12,4$

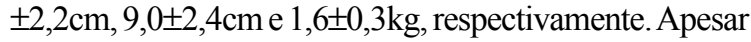
da irregularidade apresentada nas dimensões e na massa dos frutos, os resultados estão de acordo com os obtidos por SILVA et al. (1997), que reportaram frutos em média com comprimento de $15 \mathrm{~cm}$, diâmetros de $10 \mathrm{~cm}$ e massa de $1,5 \mathrm{~kg}$. Após o descascamento, obteve-se rendimento de $71,4 \%$ de frutilhos minimamente processáveis.

$\mathrm{Na}$ maioria das amostras, verificaram-se efeitos lineares do tempo de armazenamento sobre a perda de massa fresca, com excessão das amostras com laminado e PVC mais ácido ascórbico, nas quais observaram-se efeitos quadráticos significativos (Tabela 1). Verificou-se, após o segundo dia de armazenamento uma maior perda de massa fresca das amostras de frutilhos de araticum embalados com PVC, em comparação com as demais amostras. Do quarto ao décimo dia, as maiores perdas ocorreram nas amostras com PVC, seguidas por aquelas com PE e laminado (Tabela 1).

A perda de massa fresca é resultado do processo de transpiração, em conseqüência do déficit de pressão de vapor (DPV) existente entre os tecidos internos das frutas e o ambiente externo. A utilização de embalagem com filmes plásticos, durante o armazenamento refrigerado, promove maior umidade relativa ao redor das frutas, reduzindo o DPV e, conseqüentemente, a transpiração. Como conseqüência, as frutas permanecem com maior

Tabela 1 - Perda de massa fresca - PMF (\%) e pH de araticum minimamente processado, embalado com policloreto de vinila (PVC), polietileno de baixa densidade (PE) e laminado (PE/nylon/PE), com e sem ácido ascórbico (aa), e armazenado a $11^{\circ} \mathrm{C}$.

\begin{tabular}{|c|c|c|c|c|c|c|c|c|c|c|}
\hline \multirow{3}{*}{ Tratamento } & \multicolumn{5}{|c|}{ Perda de massa fresca } & \multicolumn{5}{|c|}{$\mathrm{pH}$} \\
\hline & \multicolumn{5}{|c|}{ Dias após Processamento } & \multicolumn{5}{|c|}{ Dias após processamento } \\
\hline & 2 & 4 & 6 & 8 & 10 & 2 & 4 & 6 & 8 & 10 \\
\hline $\mathrm{PVC}^{1}$ & $0,74^{\mathrm{B}}$ & $1,74^{\mathrm{C}}$ & $2,79^{\mathrm{C}}$ & $4,17^{\mathrm{C}}$ & $5,73^{\mathrm{C}}$ & $4,9^{\mathrm{A}}$ & $4,8^{\mathrm{A}}$ & $4,8^{\mathrm{A}}$ & $4,4^{\mathrm{A}}$ & $4,3^{\mathrm{BA}}$ \\
\hline $\mathrm{PE}^{2}$ & $0,04^{\mathrm{A}}$ & $0,08^{\mathrm{B}}$ & $0,19^{\mathrm{B}}$ & $0,69^{\mathrm{B}}$ & $1,36^{\mathrm{B}}$ & $5,4^{\mathrm{B}}$ & $5,2^{\mathrm{B}}$ & $5,1^{\mathrm{B}}$ & $4,8^{\mathrm{B}}$ & $4,7^{\mathrm{BC}}$ \\
\hline Laminado $^{3}$ & $0,00^{\mathrm{A}}$ & $0,01^{\mathrm{A}}$ & $0,04^{\mathrm{A}}$ & $0,12^{\mathrm{A}}$ & $0,29^{\mathrm{A}}$ & $4,8^{\mathrm{A}}$ & $4,8^{\mathrm{A}}$ & $4,9^{\mathrm{A}}$ & $4,9^{\mathrm{B}}$ & $4,9^{\mathrm{C}}$ \\
\hline $\mathrm{PVC}+\mathrm{aa}^{4}$ & $0,59^{\mathrm{B}}$ & $1,56^{\mathrm{C}}$ & $2,67^{\mathrm{C}}$ & $4,14^{\mathrm{C}}$ & $5,91^{\mathrm{C}}$ & $4,9^{\mathrm{A}}$ & $4,8^{\mathrm{A}}$ & $4,8^{\mathrm{A}}$ & $4,4^{\mathrm{A}}$ & $4,2^{\mathrm{A}}$ \\
\hline $\mathrm{PE}+\mathrm{aa}^{5}$ & $0,02^{\mathrm{A}}$ & $0,09^{\mathrm{B}}$ & $0,28^{\mathrm{B}}$ & $0,95^{\mathrm{B}}$ & $1,43^{\mathrm{B}}$ & $4,9^{\mathrm{A}}$ & $4,8^{\mathrm{A}}$ & $4,9^{\mathrm{A}}$ & $4,8^{\mathrm{B}}$ & $4,7^{\mathrm{BC}}$ \\
\hline Laminado $+\mathrm{aa}^{6}$ & $0,00^{\mathrm{A}}$ & $0,01^{\mathrm{A}}$ & $0,01^{\mathrm{A}}$ & $0,08^{\mathrm{A}}$ & $0,15^{\mathrm{A}}$ & $4,9^{\mathrm{A}}$ & $4,8^{\mathrm{A}}$ & $4,8^{\mathrm{A}}$ & $4,8^{\mathrm{B}}$ & $4,6^{\mathrm{BA}}$ \\
\hline
\end{tabular}

Os resultados para a PMF foram transformados para aplicação da análise de regressão e do teste de Tukey, utilizando-se a função: y = arco sen $(\mathrm{z} / 100)^{1 / 2}$, em que $\mathrm{z}$ é a PMF $(\%)$.

As letras sobrescritas distintas em cada coluna indicam a diferença estatística a 5\% de significância entre as médias dos tratamentos (teste de Tukey).

${ }^{1}$ PMF: $y=0,0503+0,0194 x, r^{2}=0,9758$ e $p H: y=4,9067+0,0167 x-0,0083 x^{2}, r^{2}=0,9016$.

${ }^{2} \mathrm{PMF}: \mathrm{y}=0,0190-0,0029 \mathrm{x}+0,0013 \mathrm{x}^{2}, \mathrm{r}^{2}=0,9468 \mathrm{e} \mathrm{pH}: \mathrm{y}=5,5967-0,0950 \mathrm{x}, \mathrm{r}^{2}=0,8809$.

${ }^{3}$ PMF: $y=-0,0057+0,0016 x+0,0004 x^{2}, r^{2}=0,9276$ e $p H: y=4,7500+0,0183 x, r^{2}=0,5307$.

${ }^{4}$ PMF: $y=0,0378+0,0209 x, r^{2}=0,9925$ e $p H: y=4,9133+0,0298 x-0,0100 x^{2}, r^{2}=0,8054$.

${ }^{5}$ PMF: $y=-0,0205+0,0139 x, r^{2}=0,9632$ e pH: $y=4,8067+0,0338 x-0,0040 x^{2}, r^{2}=0,5036$.

${ }^{6}$ PMF: $y=-0,0076+0,0034 x, r^{2}=0,4073$ e $p H: y=4,7733+0,4810 x-0,0060 x^{2}, r^{2}=0,4952$.

$\mathrm{y}=\mathrm{PMF}$ transformada ou $\mathrm{pH}$ e $\mathrm{x}=$ período de armazenamento (dias) em cada tratamento.

Ciência Rural, v.37, n.6, nov-dez, 2007. 
turgidez e sem sintoma de murchamento (WOODS, 1990). Esse comportamento, provavelmente, deve-se à diferença de permeabilidade dos materiais utilizados como embalagem. O PVC é mais permeável ao vapor de água que o polietileno, e este mais que o laminado (GORRIS \& PEPPELENBOS, 1992).

Em Annona cherimóia armazenada sob refrigeração a aproximadamente $12^{\circ} \mathrm{C}$, foi observado que os frutos embalados com filme de polietileno coextrusado com zeolite apresentaram perda de massa fresca próxima de $0,3 \%$ no período de quatro semanas (MELO et al., 2002). Enquanto isso, no presente estudo, com araticum minimamente processado, aos dez dias de armazenamento, observaram-se valores de: 0,15 a $0,29 \%$ de perda de massa fresca nas amostras com laminando; 1,43 a 1,36\% nas com PE e entre 5,73 e 5,91\% com PVC (Tabela 1).

Com o objetivo de verificar a eficiência do filme plástico, associado a diferentes temperaturas de armazenamento, no prolongamento da vida útil póscolheita de pinha (Anona squamosa), GUIMAR ÃES et al. (2002) observaram um aumento progressivo da perda de peso no período experimental, independentemente da atmosfera testada. Ao final de 18 dias, os frutos embalados em PVC apresentaram taxas de perda de massa fresca de 1,79, 2,50 e 4,25\%, respectivamente para os frutos submetidos às temperaturas de $10^{\circ} \mathrm{C}, 12^{\circ} \mathrm{C}$ e $16^{\circ} \mathrm{C}$. Enquanto isso, os frutos mantidos sob atmosfera ambiente perderam $10,49,16,37$ e $18,62 \%$ de massa fresca, respectivamente nas temperaturas de $10^{\circ} \mathrm{C}, 12^{\circ} \mathrm{Ce} 16^{\circ} \mathrm{C}$.

Durante o armazenamento, os frutos podem ser acondicionados em filmes plásticos, que reduzem sensivelmente a perda de massa dos frutos, retardando o amadurecimento e a elevação das taxas respiratórias, assim como reduzem a produção do etileno e atrasam o amolecimento (perda da firmeza) e várias outras transformações bioquímicas (ZAGORY \& KADER, 1988).

$\mathrm{Na}$ maioria das amostras, verificaram-se efeitos quadráticos significativos do tempo de armazenamento sobre o $\mathrm{pH}$, menos nas amostras com PE e laminado, nas quais observaram-se efeitos lineares (Tabela 1). $\mathrm{O} \mathrm{pH}$ da polpa dos frutilhos de araticum minimamente processado variou entre 4,2 e 5,4, compatível com o valor 4,6 para polpa de araticum obtido por ALMEIDA (1998). Até o sexto dia, as médias dos valores de $\mathrm{pH}$ das amostras com PE sem ácido ascórbico foram menores em relação às médias das demais. A partir do oitavo dia, as médias de $\mathrm{pH}$ das amostras com laminado e PE, com e sem ácido ascórbico, não diferiram significativamente entre si, mas sim em relação às médias das amostras com $\mathrm{PVC}$, sendo que nessas ocorreram maiores decréscimos de $\mathrm{pH}$.
Percebe-se também que a maioria das amostras sofreram decréscimo do $\mathrm{pH}$ durante o armazenamento, tornandose mais ácidas, com excessão das embaladas com laminado, nas quais quase não ocorreu variação. Resultados semelhantes foram obtidos por SARZI \& DURIGAN (2002) com abacaxis da variedade Pérola, que, em condições semelhantes, tiveram o mesmo comportamento, ou seja, diminuição no pH com conseqüente aumento na acidez titulável.

De acordo com a tabela 2, as amostras com PVC e PE, com e sem ácido ascórbico, apresentaram frutilhos atacados por microrganismos a partir do sexto dia de armazenamento. Apesar de não diferirem significativamente das outras amostras aos seis dias, essa presença torna o produto inaceitável comercialmente. Além do aspecto visual, um odor desagradável também foi percebido no quinto dia.

A influência da temperatura de estocagem em pinhas (Anona squamosa) armazenadas sob refrigeração foi verificada por ALMEIDA et al. (2002), que concluíram que os frutos armazenados à temperatura de $12^{\circ} \mathrm{C}$ mantiveram as suas características próprias para o consumo até o $9^{\circ}$ dia, tendo, a partir desse ponto, perdido a sua qualidade devido ao ataque de fungos causadores de podridões.

Neste estudo, as amostras de frutilhos embalados em laminado com ou sem ácido ascórbico não apresentaram ataque por microrganismos durante todo o período de armazenamento. Isso porque, o uso de embalagens sob vácuo parcial modifica a atmosfera de conservação do produto, diminuindo a concentração de oxigênio que, consequentemente, inibe o desenvolvimento de bolores responsáveis pela deterioração das frutas (WILEY, 1994).

Em todas as amostras, o tempo de armazenamento não afetou significativamente o teor de sólidos solúveis (Tabela 2). Ocorreu uma oscilação no teor de SS durante o armazenamento dentro de cada tratamento, provavelmente devido ao uso de frutas provenientes de diferentes plantas. Segundo SILVA et al. (2001), existe grande variação físico-química entre frutos de diferentes araticunzeiros e até entre frutos de uma mesma planta.

$\mathrm{O}$ teor de SS do araticum minimamente processado variou entre $17,6^{\circ}$ Brix e $24,3^{\circ}$ Brix (Tabela 2 ), bem acima do valor de $14,0^{\circ}$ Brix obtido por ALMEIDA (1998), provavelmente em função das condições edafoclimáticas das diferentes regiões de origem. O teor de SS das amostras com PVC e ácido ascórbico durante quase todo o período de armazenamento foi menor em relação às demais, excetuando-se no oitavo dia, quando não ocorreu diferença significativa (Tabela 2). 
Tabela 2 - Percentagem de frutilhos atacados por microrganismos - PFA e teor de sólidos solúveis - SS ( ${ }^{\circ}$ Brix) de araticum minimamente processado, embalado com policloreto de vinila (PVC), polietileno de baixa densidade (PE) e laminado (PE/nylon/PE), com e sem ácido ascórbico (aa), e armazenado a $11^{\circ} \mathrm{C}$.

\begin{tabular}{|c|c|c|c|c|c|c|c|c|c|c|}
\hline \multirow{3}{*}{ Tratamento } & \multicolumn{5}{|c|}{ Percentual de frutilhos atacados } & \multicolumn{5}{|c|}{ Teor de sólidos solúveis } \\
\hline & \multicolumn{5}{|c|}{ Dias após processamento } & \multicolumn{5}{|c|}{ Dias após processamento } \\
\hline & 2 & 4 & 6 & 8 & 10 & 2 & 4 & 6 & 8 & 10 \\
\hline $\mathrm{PVC}^{1}$ & $0,0^{\mathrm{A}}$ & $0,0^{\mathrm{A}}$ & $3,0^{\mathrm{A}}$ & $120^{\mathrm{BA}}$ & $43,0^{\mathrm{BA}}$ & $227^{\mathrm{B}}$ & $23,0^{\mathrm{CC}}$ & $223^{\mathrm{BB}}$ & $23,3^{\mathrm{A}}$ & $23,7^{\mathrm{BA}}$ \\
\hline $\mathrm{PE}^{2}$ & $0,0^{\mathrm{A}}$ & $0,0^{\mathrm{A}}$ & $0,0^{\mathrm{A}}$ & $10,0^{\mathrm{BA}}$ & $37,0^{\mathrm{BA}}$ & $24,3^{\mathrm{C}}$ & $23,7^{\mathrm{D}}$ & $223^{\mathrm{BB}}$ & $22,3^{\mathrm{A}}$ & $227^{\mathrm{BA}}$ \\
\hline Laminado $^{3}$ & $0,0^{\mathrm{A}}$ & $0,0^{\mathrm{A}}$ & $0,0^{\mathrm{A}}$ & $0,0^{\mathrm{A}}$ & $0,0^{\mathrm{A}}$ & $223^{\mathrm{B}}$ & $20,7^{\mathrm{BA}}$ & $21,7^{\mathrm{B}}$ & $22,7^{\mathrm{A}}$ & $23,7^{\mathrm{BA}}$ \\
\hline $\mathrm{PVC}+\mathrm{aa}^{4}$ & $0,0^{\mathrm{A}}$ & $0,0^{\mathrm{A}}$ & $3,0^{\mathrm{A}}$ & $40,0^{\mathrm{B}}$ & $100,0^{B}$ & $17,7^{\mathrm{A}}$ & $22,7^{\mathrm{C}}$ & $18,7^{\mathrm{A}}$ & $20,7^{\mathrm{A}}$ & $20,0^{\mathrm{A}}$ \\
\hline $\mathrm{PE}+\mathrm{aa}^{5}$ & $0,0^{\mathrm{A}}$ & $0,0^{\mathrm{A}}$ & $3,0^{\mathrm{A}}$ & $17,0^{\mathrm{BA}}$ & $40,0^{\mathrm{BA}}$ & $203^{\mathrm{BA}}$ & $22,0^{\mathrm{B}}$ & $19,7^{\mathrm{BA}}$ & $20,3^{\mathrm{A}}$ & $21,0^{\mathrm{BA}}$ \\
\hline Laminado $+\mathrm{aa}^{6}$ & $0,0^{\mathrm{A}}$ & $0,0^{\mathrm{A}}$ & $0,0^{\mathrm{A}}$ & $0,0^{\mathrm{A}}$ & $0,0^{\mathrm{A}}$ & $24,3^{\mathrm{C}}$ & $19,7^{\mathrm{A}}$ & $23,0^{\mathrm{C}}$ & $23,7^{\mathrm{A}}$ & $24,3^{\mathrm{B}}$ \\
\hline
\end{tabular}

Obs.: Os resultados para percentagem de frutilhos atacados por microrganismos foram transformados para aplicação da análise de regressão e teste de Tukey, utilizando-se a função: $y=\operatorname{arco~sen~}(z / 100)^{1 / 2}$, em que $\mathrm{z}$ é a percentagem de frutilhos atacados por microrganismos.

As letras sobrescritas distintas em cada coluna indicam a diferença estatística a 5\% de significância entre as médias dos tratamentos (teste de Tukey).

${ }^{1}$ PFA: $y=0,0125-0,0087 x+0,0014 x^{2}, r^{2}=0,7014$ e SS: regressão não significativa.

${ }^{2}$ PFA: $y=0,0207-0,0125 x+0,0160 x^{2}, r^{2}=0,5659$ e SS: regressão não significativa.

${ }^{3}$ PFA: regressão não significativa e TSS: regressão não significativa.

${ }^{4}$ PFA: $y=0,0263-0,0144 x+0,0023 x^{2}, r^{2}=0,8817$ e SS: regressão não significativa.

${ }^{5}$ PFA: $y=0,0089-0,0053 x+0,0010 x^{2}, r^{2}=0,889$ e SS: regressão não significativa.

${ }^{6}$ PFA: $y=0,0089-0,0053 x+0,0010 x^{2}, r^{2}=0,889$ e SS: regressão não significativa.

$\mathrm{y}=$ PFA transformada ou SS e $\mathrm{x}=$ período de armazenamento (dias) em cada tratamento.

Na maioria das amostras, verificaram-se efeitos quadráticos significativos do tempo de armazenamento sobre a acidez titulável, menos nas embaladas com laminado e PVC mais ácido ascórbico, nos quais observaram-se somente efeitos lineares
(Tabela 3). Durante o armazenamento, todas as amostras sofreram elevação da AT. Elevação também observada nas mesmas condições por BONNAS et al. (2003) com abacaxi minimamente processado. À medida que o fruto se aproxima da maturidade, há um

Tabela 3 - Acidez titulável (mL por g de polpa) - AT e relação entre o teor de sólidos solúveis e a acidez titulável (SS/AT) de araticum minimamente processado, embalado com policloreto de vinila (PVC), polietileno de baixa densidade (PE) e laminado (PE/nylon/PE), com e sem ácido ascórbico (aa), e armazenado a $11^{\circ} \mathrm{C}$.

\begin{tabular}{|c|c|c|c|c|c|c|c|c|c|c|}
\hline \multirow{3}{*}{ Tratamento } & \multicolumn{5}{|c|}{ AT } & \multicolumn{5}{|c|}{$\frac{S S}{A T}$} \\
\hline & \multicolumn{5}{|c|}{ Dias após processamento } & \multicolumn{5}{|c|}{ Dias após processamento } \\
\hline & 2 & 4 & 6 & 8 & 10 & 2 & 4 & 6 & 8 & 10 \\
\hline $\mathrm{PVC}^{1}$ & $3,1^{\mathrm{C}}$ & $3,1^{\mathrm{BC}}$ & $3,8^{\mathrm{C}}$ & $4,6^{\mathrm{C}}$ & $5,1^{\mathrm{C}}$ & $7,2^{\mathrm{BA}}$ & $7,3^{\mathrm{A}}$ & $5,9^{\mathrm{BA}}$ & $5,1^{\mathrm{A}}$ & $4,6^{\mathrm{A}}$ \\
\hline $\mathrm{PE}^{2}$ & $2,5^{\mathrm{BA}}$ & $2,9^{\mathrm{A}}$ & $3,0^{\mathrm{A}}$ & $3,8^{\mathrm{BA}}$ & $4,2^{\mathrm{BA}}$ & $9,5^{\mathrm{C}}$ & $8,1^{\mathrm{B}}$ & $7,3^{\mathrm{DC}}$ & $5,9^{\mathrm{A}}$ & $5,4^{\mathrm{BA}}$ \\
\hline Laminado $^{3}$ & $2,8^{\mathrm{B}}$ & $2,8^{\mathrm{A}}$ & $3,2^{\mathrm{BA}}$ & $3,4^{\mathrm{A}}$ & $3,5^{\mathrm{A}}$ & $7,8^{\mathrm{B}}$ & $7,3^{\mathrm{A}}$ & $6,7^{\mathrm{CB}}$ & $6,7^{\mathrm{A}}$ & $6,7^{\mathrm{B}}$ \\
\hline $\mathrm{PVC}+\mathrm{aa}^{4}$ & $2,7^{\mathrm{BA}}$ & $3,3^{\mathrm{C}}$ & $3,5^{\mathrm{BC}}$ & $4,2^{\mathrm{CB}}$ & $4,8^{\mathrm{CB}}$ & $6,5^{\mathrm{A}}$ & $6,9^{\mathrm{A}}$ & $5,3^{\mathrm{A}}$ & $5,0^{\mathrm{A}}$ & $4,2^{\mathrm{A}}$ \\
\hline $\mathrm{PE}+\mathrm{aa}^{5}$ & $2,5^{\mathrm{A}}$ & $3,0^{\mathrm{BA}}$ & $2,9^{\mathrm{A}}$ & $3,3^{\mathrm{C}}$ & $43^{\mathrm{CBA}}$ & $8,1^{\mathrm{B}}$ & $7,3^{\mathrm{A}}$ & $6,7^{\mathrm{DB}}$ & $6,1^{\mathrm{A}}$ & $4,9^{\mathrm{A}}$ \\
\hline Laminado $+\mathrm{aa}^{6}$ & $3,0^{\mathrm{C}}$ & $2,9^{\mathrm{A}}$ & $2,8^{\mathrm{A}}$ & $3,3^{\mathrm{A}}$ & $3,8^{\mathrm{A}}$ & $8,0^{\mathrm{B}}$ & $6,8^{\mathrm{A}}$ & $8,0^{\mathrm{D}}$ & $7,1^{\mathrm{A}}$ & $6,4^{\mathrm{B}}$ \\
\hline
\end{tabular}

As letras sobrescritas distintas em cada coluna indicam a diferença estatística a 5\% de significância entre as médias dos tratamentos (teste de Tukey).

${ }^{1} \mathrm{AT}: \mathrm{y}=2,9467+0,0040 \mathrm{x}+0,0220 \mathrm{x}^{2}, \mathrm{r}^{2}=0,9462$ e SS/AT: $\mathrm{y}=8,2167-0,3650 \mathrm{x}, \mathrm{r}^{2}=0,8455$.

${ }^{2} \mathrm{AT}: \mathrm{y}=2,3733+0,0638 \mathrm{x}+0,0119 \mathrm{x}^{2}, \mathrm{r}^{2}=0,9152$ e SS/AT: $\mathrm{y}=10,3500-0,5183 \mathrm{x}, \mathrm{r}^{2}=08902$.

${ }^{3} \mathrm{AT}: \mathrm{y}=2,5400+0,1000 \mathrm{x}, \mathrm{r}^{2}=0,7916$ e SS/AT: $\mathrm{y}=8,7060-0,4970 \mathrm{x}+0,0298 \mathrm{x}^{2}, \mathrm{r}^{2}=0,4805$.

${ }^{4}$ AT: $y=2,2033+0,2483 x, r^{2}=0,8822$ e SS/AT: $y=6,9867-0,0980 x-0,0190 x^{2}, r^{2}=0,7070$.

${ }^{5} \mathrm{AT}: \mathrm{y}=2,7733-0,1230 \mathrm{x}+0,0260 \mathrm{x}^{2}, \mathrm{r}^{2}=0,8792$ e SS/AT: $\mathrm{y}=8,9433-0,3850 \mathrm{x}-0,016 \mathrm{x}^{2}, \mathrm{r}^{2}=0,8886$

${ }^{6} \mathrm{AT}: \mathrm{y}=3,4888-0,2862 \mathrm{x}+0,0319 \mathrm{x}^{2}, \mathrm{r}^{2}=8873$ e SS/AT: $\mathrm{y}=8,1167-0,1417 \mathrm{x}, \mathrm{r}^{2}=0,3147$

$\mathrm{y}=\mathrm{AT}$ ou SS/AT e $\mathrm{x}=$ período de armazenamento (dias) em cada tratamento.

Ciência Rural, v.37, n.6, nov-dez, 2007. 
característico aumento da taxa respiratória, da síntese do etileno, da degradação da parede celular e da síntese dos açúcares a partir das reservas. Neste trabalho, o $\mathrm{pH}$ diminuiu e, conseqüentemente, a acidez aumentou, provavelmente devido à fermentação dos açúcares com formação de ácidos.

Durante o armazenamento, os valores obtidos para a AT dos frutilhos de araticum minimamente processado variaram entre 2,5 e 5,1 $\mathrm{mL}$ de $\mathrm{NaOH} 0,1 \mathrm{~N}$ por g de polpa (Tabela 3 ), enquanto que ALMEIDA (1998) obteve valores de acidez titulável para a polpa de araticum ao redor de $5,5 \mathrm{~mL}$ de $\mathrm{NaOH}$ $0,1 \mathrm{~N}$ por g de polpa. Nota-se, na tabela 3 , que, no segundo dia de armazenamento, as amostras com PVC sem ácido ascórbico e as amostras com laminado e ácido ascórbico apresentaram maior acidez em relação às demais. A partir do quarto dia, as com PVC não diferiram significativamente entre si, mas apresentaram uma acidez maior em relação a praticamente quase todas as outras amostras (exceto as com PE com ou sem ácido ascórbico). Esta tendência manteve-se até o décimo dia, quando as amostras com laminado não diferiram significativamente entre si e em relação às amostras com PE, apresentando os menores valores de acidez titulável.

Verificaram-se efeitos quadráticos significativos do tempo de armazenamento sobre a relação SS/AT nas amostras com laminado, PVC mais ácido ascórbico e PE mais ácido ascórbico, enquanto que nas demais observaram-se somente efeitos lineares (Tabela 3). Neste trabalho, ocorreu uma tendência geral de diminuição da relação SS/AT durante o decorrer do experimento (Tabela 3 ).

Os frutilhos de araticuns de todas as amostras apresentavam-se com cor viva e aparência muito boa no primeiro dia de armazenamento refrigerado. Os embalados com PVC e PE, com ou sem ácido ascórbico, apresentavam-se com início de escurecimento e aparência regular no terceiro dia e escurecidos e com aparência péssima a partir do quinto dia. Não houve diferença de aparência entre essas amostras durante todo o período estudado. A partir do terceiro dia, as amostras com PVC e PE apresentaram aparência inferior em relação às amostras com laminado, mantendo essa tendência até o final do armazenamento. No primeiro dia, as amostras com laminado apresentavam aparência muito boa, com cor viva. Contudo, houve uma constante queda na qualidade, passando a uma aparência boa com coloração opaca do terceiro ao sétimo dia, e apresentando aparência regular com início de escurecimento no nono dia, que se manteve até o final do experimento (Tabela 4). Provavelmente devido à baixa concentração de oxigênio na atmosfera de armazenamento (vácuo parcial) e à baixa permeabilidade do laminado a esse gás, as reações de escurecimento foram retardadas.

Avaliando a aparência de frutos de Annona cherimoia, MELO et al. (2002) observaram o início do escurecimento a partir da segunda semana de armazenamento, o que depreciou sensivelmente os frutos para a comercialização. MARTINEZ et al. (1993), também estudando o armazenamento de frutos de Annona cherimoia, observaram que, por se tratar de uma fruta climatérica, as mudanças que causam a perda de firmeza e o escurecimento devem-se à rápida elevação da taxa de biossíntese de etileno no início do processo de amadurecimento. $\mathrm{O}$ aumento nas taxas da atividade respiratória nas anonáceas é seguido de rápida modificação na composição química, tornando o sabor e o aroma muito agradáveis; em contrapartida, há um decréscimo muito rápido da firmeza da polpa (ALVES et al., 1997).

Em frutas e hortaliças minimamente processadas, existem vários tipos de reações oxidativas que causam o escurecimento do tecido (WILEY, 1994). O uso de aditivos para a preservação da coloração desses produtos tem sido alvo de estudo de vários pesquisadores. LAMIKANRA \& WATSON (2000) verificaram que a utilização de ácido ascórbico preservou a coloração de melão cantaloupe minimamente processado por 25 dias, a $4^{\circ} \mathrm{C}$. Porém, neste experimento, esse efeito não foi observado, provavelmente em função da dose ou do método de aplicação do ácido ascórbico. No trabalho citado, os

Tabela 4 - Nota de aparência de araticum minimamente processado, embalado e armazenado a $11^{\circ} \mathrm{C}$.

\begin{tabular}{lcccccc}
\hline \multirow{2}{*}{ Tratamento } & \multicolumn{6}{c}{ Dias após Processamento } \\
\cline { 2 - 7 } & 1 dia & 3 dias & 5 dias & 7 dias & 9 dias & 11 dias \\
\hline PVC & $1,00^{\mathrm{A}}$ & $3,00^{\mathrm{B}}$ & $3,33^{\mathrm{B}}$ & $3,67^{\mathrm{B}}$ & $4,00^{\mathrm{B}}$ & $4,00^{\mathrm{B}}$ \\
PE & $1,00^{\mathrm{A}}$ & $3,00^{\mathrm{B}}$ & $3,33^{\mathrm{B}}$ & $4,00^{\mathrm{B}}$ & $4,00^{\mathrm{B}}$ & $4,00^{\mathrm{B}}$ \\
Laminado & $1,00^{\mathrm{A}}$ & $2,00^{\mathrm{A}}$ & $2,00^{\mathrm{A}}$ & $2,00^{\mathrm{A}}$ & $3,00^{\mathrm{A}}$ & $3,00^{\mathrm{A}}$ \\
PVC + aa & $1,00^{\mathrm{A}}$ & $3,00^{\mathrm{B}}$ & $3,33^{\mathrm{B}}$ & $4,00^{\mathrm{B}}$ & $4,00^{\mathrm{B}}$ & $4,00^{\mathrm{B}}$ \\
PE + aa & $1,00^{\mathrm{A}}$ & $3,00^{\mathrm{B}}$ & $3,33^{\mathrm{B}}$ & $4,00^{\mathrm{B}}$ & $4,00^{\mathrm{B}}$ & $4,00^{\mathrm{B}}$ \\
Lamininado + aa & $1,00^{\mathrm{A}}$ & $2,00^{\mathrm{A}}$ & $2,00^{\mathrm{A}}$ & $2,00^{\mathrm{A}}$ & $3,00^{\mathrm{A}}$ & $3,00^{\mathrm{A}}$ \\
\hline
\end{tabular}

As letras sobrescritas distintas em cada coluna indicam a diferença estatística a $5 \%$ de significância entre as médias dos tratamentos (teste de comparação de médias nos ensaios inteiramente casualisados e teste Kruskal-Wallis /estatística não paramétrica);

O tempo de armazenamento afeta a aparência ao nível de 5\% de significância nas amostra com PVC e PE, com ou sem ácido ascórbico e não afeta as amostras embaladas com laminado, com ou sem ácido ascórbico, segundo o teste de Kruskal-Wallis. 
pedaços de melão foram imersos em solução de ácido ascórbico; mas, no caso do araticum, devido à sua textura mucilaginosa, este método não foi utilizado em função da facilidade de perda da forma e da massa dos frutilhos na imersão.

\section{CONCLUSÕES}

Sob as condições experimentais estabelecidas neste trabalho, conclui-se que: o ácido ascórbico não evitou o escurecimento do araticum minimamente processado; independentemente do tipo de embalagem e do uso ou não de aditivos, ocorreu redução do $\mathrm{pH}$ e aumento na acidez titulável e da relação sólidos solúveis/acidez total do produto ao decorrer do período de armazenamento; o laminado a vácuo foi a embalagem que melhor preservou a aparência do produto, sem ataque aparente de microrganismos, mantendo-a boa até o sétimo dia de armazenamento, enquanto que as demais embalagens apresentaram frutos com aparência imprópria para a comercialização a partir do terceito dia de armazenamento.

\section{REFERÊNCIAS}

ALMEIDA, R.F. et al. Armazenamento refrigerado de pinha (Anona squamosa). In: CONGRESSO BRASILEIRO DE FRUTICULTURA, 17., 2002, Belém. Anais... Belém: SBF, 2002. 1 CD-ROM

ALMEIDA, S.P. de. Frutas nativas do cerrado: caracterização físico-química e fonte potencial de nutrientes. In: SANO, S. M.; ALMEIDA, S. P. (Org.). Cerrado ambiente e flora. Planaltina: EMBRAPA-CPAC, 1998. p.247-285.

ALVES, R.E. et al. Colheita e pós-colheita de anonáceas. In: SÃO JOSÉ, A.R. et al. (Org). Anonáceas: produção e mercado (pinha, graviola, atemóia e cherimólia). Vitória da Conquista: DFZ/UESB, 1997. p.240-256.

ARAÚJO, J.M.A. Química de alimentos. Viçosa: UFV, 2001. $411 \mathrm{p}$

BALDWIN, E.A. et al. Edible coatings for lightly processed fruits and vegetables. Hortscience, Alexandria, v.30, n.1, p.3538, 1987.

BONNAS, D.S. et al. Qualidade do abacaxi cv. smooth cayenne minimanente processado. Revista Brasileira de Fruticultura, Jaboticabal, v.25, n.2, p.206-209, 2003

CHITARRA, M.I.F. Processamento mínimo de frutos e hortaliças. Viçosa: Centro de Produções Técnicas, 1998. 112p.

CHITARRA, M.I.F.; CHITARRA, A.B. Pós-colheita de frutos e hortaliças: fisiologia e manuseio. 2.ed. Lavras: UFLA, 2005. 783p.

GOMES, F.P. Curso de estatística experimental. 11.ed. São Paulo: Nobel, 1985. 466p.

GORRIS, L.G.M.; PEPPELENBOS, H.W. Modified atmosphere and vacuum packaging to extend the shelf life of respiring food products. HortTechnology, Alexandria, v.2, n.3, p.303-309, 1992.

GUIMARÃES, E.F.P. et al. Uso de atmosfera modificada e refrigeração no prolongamento da vida útil pós-colheita de pinha (Annona squamosa L.). In: CONGRESSO BRASILEIRO DE FRUTICULTURA, 17., 2002, Belém. Anais... Jaboticabal: SBF, 2002. 01 CD-ROM.

INSTITUTO ADOLFO LUTZ. Normas analíticas do Instituto Adolfo Lutz. Métodos químicos e físicos para análise de alimentos. 3.ed. São Paulo, 1985. 533p.

LAMIKANRA, O.; WATSON, M.A. Cantaloupe melon peroxidase: characterization and effects of additivies on activity. Nahrung, Weinheim, v.44, n.3, p.168-172, 2000

MARTINEZ, G. et al. Ethylene biosynthesis and physicochemical changes during fruit ripening of cherimoya (Annona cherimola Mill.) fruit. Journal of Horticultural Science, London, v.68, n.4, p.477-483, 1993.

MELO, M.R. et al. Conservação refrigerada de cherimóia embalada em filme plástico com zeolite. Bragantia, Campinas, v.61, n.1, p.71-76, 2002

RIBEIRO J.F. et al. Araticum. Jaboticabal: FUNEP/SBF, 2000. 52p. (Frutas Nativas, 7).

SARZI, B.; DURIGAN, J.F. A nálise física e química de produtos minimanente processados de abacaxi pérola. Revista Brasileira de Fruticultura, Jaboticabal, v.24, n.2, p.28-32, 2002.

SAS - SAS INSTITUTE. The SAS system for Windows, 6.12. Carry, 1996. 10 CD-ROM.

SILVA, D.B. da et al. Frutos do cerrado. Brasília: EMBRAPACPAC, 2001. 178p.

SILVA, J.A. et al. Coleta de sementes, produção de mudas e plantio de espécies frutíferas nativas dos cerrados: informações exploratórias. Brasília: EMBRAPA-CPAC, 1997. $23 \mathrm{p}$.

UNDURRAGA, P.M. et al. Identificación y caracterización de desordenes fisiológicos en chirimoyas (Annona cherimola Mill.) cv. Concha Lisa em refrigeración. In: CONGRESO INTERNACIONAL DE ANONÁCEAS, 1997, Chapingo. Memorias... Chapingo: Universidad Autonoma de Chapingo, 1997. p.277-291

WILEY, R.C. Minimally processed refrigerated fruits and vegetables. New York: Chapman \& Hall, 1994. 368p.

WOODS, J.L. Moisture loss from fruits and vegetables. Postharvest News and Information, Wallingford, v.1, n.3, p.195-199, 1990.

ZAGORY, D.; KADER, A.A. Modified atmosphere packaging of fresh produce. Food Technology, Chicago,v.42, n.9, p.7077, 1988. 